\title{
Tactical Planning Models for Supply Chain Management*
}

\author{
Jayashankar M. Swaminathan \\ The Kenan-Flagler Business School \\ University of North Carolina \\ Chapel Hill, NC 27599
}

\author{
Sridhar R. Tayur \\ GSIA \\ Carnegie Mellon University \\ Pittsburgh, PA 15213
}

February 2001; Revised November 2002, January 2003

\section{Introduction}

Supply chain management includes the implementation of efficient policies related to procurement of raw material, transforming them into semi-finished and finished products and distributing them to the end customer, thereby transcending multiple business units. Poor supply chain management (more often than not) predominantly results in excessive amounts of inventory, the largest asset for many firms. Inventory is generally carried by firms to hedge against uncertainty of different types (demand, process and supply) as well as to account for economic efficiencies. The former are managed with safety stocks (either in raw material or in finished goods), and the latter through batches (lot sizes). Typically, both these types of inventories need to be considered simultaneously, as one is affected by the other. Outside the manufacturing floor, a major challenge that companies face relates to the management of safety stocks rather than with the choice of economic lot-sizes. Thus, efficient coordination of the supply chain relies heavily on how well the uncertainties related to demand, process and supply are managed. Tactical planning is the setting of key operating targets (such as safety stocks, planned lead times and batch sizes) across the different units

\footnotetext{
*Invited chapter in the Handbook of OR/MS on Supply Chain Management edited by Graves and de Kok. Comments are welcome.
} 
in a coordinated manner. These key operating targets then provide guidance as to which day-to-day operations (either in manufacturing, logistics or procurement) can be executed. While several software tools are available in the execution arena (using the more mature area of deterministic mathematical programming), effective tactical planning tools are yet to be fully developed.

Although it would be ideal from a research standpoint to develop large scale integrated models consisting of multiple entities while trying to understand effective supply chain practices, it is often very difficult (and in most cases impossible) to get any useful insights from such large models because they are intractable. As a result, researchers in the area of operations management over the past 50 years have tried to develop insights on simpler models which could then be used as building blocks to study more complex and real supply chains. The approach adopted has been one where one decomposes a multi-level supply chain - such as an assembly or a distribution system - and analyzes individual facilities with specific characteristics under different conditions. Such models will be the main focus of this chapter. For practical applications large scale models have been developed and implemented based on these building blocks. Some of these papers will be briefly reviewed at the end of this chapter (these are discussed in greater detail in chapter 14).

Before introducing the different parameters related to analysis of such models, we will introduce three important notions related to the modelling and analysis that differentiate supply chain models developed by researchers in the past. First is related to the time granularity of the model, second to time horizon of the study and the third related to performance measures. In terms of granularity, the model of analysis of any inventory system (for single or multiple facility) could be based either on a continuous basis or on a periodic basis. In models with continuous review (of inventory and other parameters), the assumption is that demand occurs continuously with a demand rate (units/time) that could be deterministic or stochastic, and costs that are incurred every instant of time. In a periodic review setting (or discrete time models), the assumptions are that demand occurs every period whose granularity could be dependent on the actual environment (say a day, a week, a month or quarter). In many real environments the review process is periodic; we will focus on such models 
called discrete-time models in this paper. Discrete time models can be developed for a single period, multiple periods or for an infinite horizon. The performance measures related to the analysis of discrete-time models could be single period costs, discounted multi-period or infinite horizon costs or average costs over the infinite horizon (the break-up of these costs will be explained later). Alternatively, for each horizon of analysis, we may specify a service level requirement. Previous research oriented books that have addressed supply chain models include Graves et. al. (1993), Tayur et. al. (1998) and Zipkin (2000).

Once a complex multi-stage, multi-product supply chain has been decomposed to its basic building block, we are left with single product, single stage (or facility) models that interact with each other through upstream and downstream parameters. The decomposition into a single product setting itself needs some care: for example, if multiple products share a certain common capacity, or if there are fixed costs in ordering a set of products, these interactions across products have to be accounted for. Once decomposed, any single facility in a supply chain faces three types of parameters - downstream parameters, upstream parameters and facility parameters.

- Downstream Parameters: Downstream parameters are those that depend on - (1) actions of the facilities downstream (such as the customers); (2) the way information obtained from downstream facilities is processed; (3) the contractual relationship with downstream facilities. In developing a supply chain model for a single facility, the main downstream parameters to be considered are as follows.

- Demand Process: The demand that gets generated at any facility depends on the operations and decisions of the downstream facilities (the customers). Seldom do we find real environments where demand is deterministic because of the uncertainties in the business environment. As a result, we will concentrate on models with stochastic demand. In a discrete time setting, the simplest stochastic demand assumption is that it is i.i.d (independent and identically distributed). This implies that in each period the demand is independent from other periods but is generated from the same distribution. Another (more realistic) related assumption is that 
of independent but non-identical demands in different periods also called non-stationary distribution. Finally, the demand process can be modelled in a more complex way in terms of being dependent as well as different in each period (auto-correlated demands are discussed in greater detail in chapter 8).

- Forecasts and Information: In many business environments, it is not possible to respond to the generated demand immediately (due to lead time for production, supply and distribution as well as capacity constraints). In such cases, the facility develops forecasts for demand in any period and utilizes that to produce "enough" to match the requirements of demand. Another way to predict the demand is to gain more information about the ordering process at the downstream facility (which generates the demand) or try to predict unknown parameters in the demand distribution using the information about realized demand until then. These predictions are utilized to develop the inventory policy for the facility.

- Contracts: Contracts with downstream facilities typically determine the costs as well service that needs to be provided by the facility. Contracts dictate whether late or partial shipments will be allowed as well as the penalty cost for stocking-out or delaying the shipment. Multi-period discrete-time models can be differentiated based on none, partial or complete backlogging of demand. In the case of no backlogging (also called lost sales), the firm loses all the demand that it fell short of in a given period whereas in the complete backlogging case, the firm is allowed to ship the remaining order in future periods (but has to incur the penalty). Other parameters in the contract could be level of service (such as fill rate constraint), quality, vendor managed inventory, end of life returns as well as delivery flexibility. Supply chain models have been developed with the fundamental objective of optimal contracts. For details see Lariviere (1998), Tsay et. al. (1998), Cachon (2002) and Chen (2002).

- Upstream Parameters: Upstream parameters depend on the decisions of the suppliers upstream related to their production process. 
- Lead Time: In most real environments, there are significant lead times involved before the material ordered is obtained from the supplier. Some suppliers are more reliable than others in that their lead times for fulfillment are more accurate and do not vary a whole lot from period to period. In extreme cases, suppliers may situate a hub near the facility in which case the lead time is negligible and can be ignored. Supply chain models can be developed with zero lead time, fixed deterministic lead time and stochastic lead time. As will be noted later, many results related to zero lead times can be extended to fixed deterministic lead times. It is also quite common today to have dual lead times, either because of multiple suppliers, or because there are multiple options on how to obtain material from the same supplier.

- Yield: Yield refers to the percentage of requested order that got delivered from the supplier. Generally, yield is modelled as a random number that represents the fraction of the order that was satisfied. Additive models of yield have also been developed. Clearly, both yield and the lead time together determine the supply process. For example, a supplier may deliver the products always in two weeks but may falter in terms of amount of delivery. On the other hand, the supplier could be delivering the exact quantities ordered but may be delivering them with different lead times.

- Facility Parameters: The performance of the facility among other factors depends on the capacity available for production, the number of products produced, setup costs and variable costs associated with production, randomness in the production process, and operational policies such as inventory decisions as well as sequencing and scheduling.

- Capacity: Most real facilities have finite capacity for production in any given period which can be increased to an extent through outsourcing on a need basis. However, incorporating capacity in to a supply chain model may make it more difficult to analyze. As a result, the earliest models assumed infinite capacities in the process and more recent models have incorporated finite fixed capacity in their analysis. 
- Costs: There are four types of costs associated with the production and inventory at the facility. First there is a per unit production cost (or variable cost of production). Second there could be a fixed cost associated with production. This cost typically reflects the costs associated with changing the setup of machines (equivalent of setup or changeover time). Third there is a per unit holding cost that is charged to inventory remaining at the facility at the end of the period. Finally, there may be a salvage cost (usually negative) which represents the salvage value of the inventory at the end of the horizon. These costs along with the the stock-out or penalty cost described above comprise the total costs incurred by the facility.

- Product and Process Characteristics: The number of products that are produced at the facility and their inter-relationships (complementary or substitutes) affect the performance of the facility. Further, the process characteristics such as yield influence the performance. As more product and process characteristics are incorporated in a single model, it becomes more difficult to obtain analytical insights. For most part of this paper, we will discuss the base case with single product, neglecting the above characteristics. However, we will discuss results related to some of the above in section 5 on extensions.

- Operational Decisions: The fundamental decision in almost all supply chain models relates to (1) how much inventory to stock in a given period and (2) when to produce/order. All the models discussed in this paper develop insights on these two fundamental questions. This is often called the inventory policy or inventory ordering policy. The inventory policy determines the operating performance of the facility. Clearly, the scheduling of different products, their lot sizes and their sequence affects the real performance in cases where there are multiple products. In most of the discrete-time models (the focus of this paper) this aspect is typically ignored.

In section 2 , we present the notation that will used throughout the chapter. In sections 3 and 4 , we discuss models with the stationary and non-stationary demand 
respectively. In section 5, we discuss various extensions - multi-level systems, multiproduct systems, multiple suppliers, random yield and perishability of products. We discuss industry applications in section 6 and conclude in section 7 .

\section{Notations Used}

In this section we will provide a list of all notations used in this paper for ease of reference.

- $h$ : per unit holding cost incurred on inventory in a period;

- $s$ : per unit salvage cost at the end of the horizon;

- $\pi$ : per unit stock-out cost on demand not satisfied in a period;

- $c$ : per unit production/ordering cost of the product;

- $K$ : set-up cost for production/ordering;

- $\xi$ : demand realized in a period;

- $\mu$ : mean value of $\xi$;

- $\sigma$ : standard deviation of $\xi$;

- $f, F$ : probability density and cumulative density functions for $\xi$.

- $x$ : beginning inventory in a period;

- $y$ : inventory level after an order has been placed;

- $J_{n}(x)$ : optimal cost to go with $n$ periods remaining in the horizon with $x$ units on hand;

- $G(y)$ : expected one-period cost given the inventory level is $y$ after ordering;

- $\alpha$ : discount rate $0<\alpha \leq 1$;

- $\delta(x)$ : threshold function where $\delta(x)=1 x>0$ and $\delta(0)=0$; 
- $C$ : capacity available in a period;

- $l$ : lead time for supply in a period;

- $p_{l}$ : probability density for lead time from the supplier equal to $l$ periods;

Any of the variables above with a subscript $t$ (such as $\xi_{t}$ or $x_{t}$ ) represent the value of the variable for the time period $t$. Similarly, any of the above variables with a $*$ in the superscript (such as $y^{*}$ ) represent the optimal value.

\section{$3 \quad$ Stationary and Independent Demand}

Stationary and independent demand models assume that the demand $\xi$ in every period comes from i.i.d distribution. With the i.i.d assumption in demand, one typically need not be concerned about the particular period $t$ one is analyzing as well as the demand history up to that period if other variables such as cost are also stationary. This simplifies the analysis and as a result we will focus on these models first. Note that an elaborate description and analysis of these models have appeared in earlier handbooks edited by Heyman and Sobel (1984) and Graves et. al. (1993).

\begin{tabular}{|l|l|l|l|}
\hline Year & Reference & Year & Reference \\
\hline 1951 & Arrow, Harris and Marschak & 1971 & Morton \\
\hline 1958 & Karlin & 1972 & Wijngaard \\
\hline 1958 & Karlin and Scarf & 1979 & Ehrhardt \\
\hline 1960 & Scarf & 1979 & Nahmias \\
\hline 1963 & Iglehart & $1986 \mathrm{ab}$ & Federgruen and Zipkin \\
\hline $1965 \mathrm{~b}$ & Veinott & 1989 & De Kok \\
\hline 1965 & Veinott and Wagner & 1991 & Zheng \\
\hline $1966 \mathrm{~b}$ & Veinott & 1991 & Zheng and Federgruen \\
\hline 1970 & Kaplan & 1993 & Tayur \\
\hline 1971 & Porteus & 1996 & Van Donselaar, De Kok and Rutten \\
\hline
\end{tabular}

Table 1: Papers on the Base Case: Single Product Single Stage Stationary and Independent Demand. 


\subsection{Single Period}

The single period stochastic inventory model deals with the problem of deciding how much to order at the beginning of the period given that demand is uncertain and there are penalty costs for lost demand and holding costs for excess inventory. This problem is also called the news vendor problem because it mimics the issue faced by a news vendor who needs to decide how many copies of a newspaper need to be purchased at the beginning of the day given that for every copy that sells there is a profit and every copy that remains at the end of the day there is a loss. Note that in the following discussions we assume cost minimization to be the objective (by assigning a penalty cost for lost demand); however, these problems can also be studied as profit maximization problems.

To begin we will assume the simplest model where there are no salvage and setup costs $(s=0, K=0)$ as well as the lead time for delivery is zero. Let $x$ be the starting inventory at the facility, then the objective is to minimize the expected costs during the period by producing/ordering enough to bring the inventory level to $y \geq x$ after ordering. We further assume that $-h<c<\pi$. Then the single period expected cost $L(y, x)$ given $x$ is

$$
L(y, x)=c(y-x)+\pi \int_{y}^{\infty}(\xi-y) d F(\xi)+h \int_{0}^{y}(y-\xi) d F(\xi)
$$

The first term represents the ordering cost while the second and third terms represent the penalty and holding costs respectively.

Let $G(y)$ be defined as follows

$$
G(y)=c y+\pi \int_{y}^{\infty}(\xi-y) d F(\xi)+h \int_{0}^{y}(y-\xi) d F(\xi)=L(y, x)+c x
$$

It is clear that $G(y)$ is convex in $y$. As a result, $L(y, x)$ is convex in $y$. The optimal value of the cost $L^{*}$ is obtained by setting the first derivative equal to 0 which gives

$$
y^{*}=F^{-1}\left(\frac{\pi-c}{\pi+h}\right)=F^{-1}\left(\frac{\pi-c}{(\pi-c)+(c+h)}\right)
$$


The ratio $\frac{\pi-c}{\pi+h}$ is called the critical fractile and the value of $y^{*}$ is called the base stock level. Since it is optimal to order or produce up to an inventory level of $y^{*}$ at the beginning of each period, such a policy is also called an order upto policy. An order up to policy orders up to $y^{*}$ if $x<y^{*}$ and does not order anything if $x \geq y^{*}$. An important thing to notice about this policy is that the order up to level $y^{*}$ is independent of the initial inventory. Another interesting point about (3) is that the critical fractile can be written as a fraction of underage costs and overage + underage costs where overage cost is the cost of having one additional unit than demanded $(c+h)$ and the underage cost is the cost having one less unit than demanded $(\pi-c)$. The above value of $y^{*}$ can also be computed by equating marginal benefits to marginal costs as given in (4).

$$
(\pi-c)(1-F(y))=(c+h) F(y)
$$

The difference between $y^{*}$ and $\mu$ (the average demand) is referred to as the buffer stock. The earliest reference of this term (as indicated by Arrow et. al. 1958) appears in Edgeworth (1888) within a banking context where the probability of running out was pre-specified. Arrow, Harris and Marschak (1951) provide the first reference of this model with underage and overage costs.

If the per unit salvage cost $s$ is included in the model, then the overage cost is equal to $c+h+s$ and the critical fractile is adjusted accordingly. If the facility has a production capacity of $C$ units in the period, then the optimal policy is as follows.

$$
y^{*}= \begin{cases}F^{-1}\left(\frac{\pi-c}{\pi+h}\right) & \text { if } x \leq F^{-1}\left(\frac{\pi-c}{\pi+h}\right) \leq x+C \\ x+C & \text { if } x+C \leq F^{-1}\left(\frac{\pi-c}{\pi+h}\right) \\ x & \text { if } x \geq F^{-1}\left(\frac{\pi-c}{\pi+h}\right)\end{cases}
$$

The policy given above is also termed as a modified base stock policy since the policy tries to get as close to the base stock level when there is capacity constraint.

\subsubsection{Setup Costs}

In the above model one could include a setup cost $K$ each time an order is placed (or production initiated). The corresponding cost function is given by (for $y \geq x$ ) 
$L(y, x)=c(y-x)+K \delta(y-x)+\pi \int_{y}^{\infty}(\xi-y) d F(\xi)+h \int_{0}^{y}(y-\xi) d F(\xi)=G(y)+K \delta(y-x)-c x$

We know that given that an order is going to be placed $(y>x)$ the function $L(y, x)$ is convex in $y$ since $G(y)$ is convex in $y$ and the value is minimized at $y^{*}=F^{-1}\left(\frac{\pi-c}{\pi+h}\right)$. The associated cost is equal to $G\left(y^{*}\right)+K-c x$. Clearly if $G(x)-c x \leq G\left(y^{*}\right)+K-c x$ or $G(x) \leq G\left(y^{*}\right)+K$ then it is not optimal to place an order. However, if $G(x)>$ $G\left(y^{*}\right)+K$ then it is optimal to order to reach $y^{*}$. Thus, with the introduction of the setup cost the optimal inventory policy has two parameters often referred to as $(s, S)$ policy where if $x<s$ then the inventory level is brought to $S$ and if $x \geq s$ then no orders are placed. In the above policy $S=y^{*}$ and $s \leq S$ is chosen in such a way so that $G(s)=G(S)+K$.

\subsubsection{General Cost Assumptions}

Thus far we have assumed that ordering, holding and shortage costs are linear. One can also have other types of functions for these costs which are non-linear and maybe concave or convex. Let us denote the holding, penalty and ordering cost functions as $\hat{h}, \hat{\pi}$ and $\hat{c}$ respectively (for now we will neglect the salvage costs). Clearly, if $\hat{h}$ and $\hat{\pi}$ are convex then $G(y)$ as defined in (2) is convex in $y$ so one can find the optimal $y^{*}$. The optimal $y^{*}$ will not be given by a simple critical fractile anymore. As indicated in Porteus (1991), the case of quadratic holding and stock-out costs (defined on the positive values of the argument) leads to an interesting result in that the optimal inventory level is equal to mean $\mu$ when overage and underage costs are identical under other standard assumptions. Note that this is different from the linear case where we stock the median value when the underage and overage costs are equal. If the holding and stock-out costs are non-linear and non-convex even then under certain conditions the optimal $y^{*}$ can be found. Those conditions are that if $G(y)$ can be written as $G(y)=A+\int_{0}^{\infty} g(y-\xi) f(\xi) d(\xi)$ where $A$ is constant and $g$ is quasi convex and $f(\xi)$ is a polya frequency function (P.F.F) of order 3 (Karlin 1958).

The model and results get somewhat changed if we have convex ordering/production costs but linear penalty and holding costs. Karlin (1958) shows that if $\hat{c}(x)$ is convex 
in $x$ and $\lim _{x \rightarrow 0} \hat{c}(x)=0$ then $y^{*}(x)$ is increasing in $x$ but $y^{*}(x)-x$ (the amount ordered) is decreasing in $x$. This is called a generalized base stock policy. The special case of piece wise linear convex costs leads to a generalized policy with finite number of distinct base stock levels. For example, the case with two piece wise linear function often results in real life when there are two suppliers and the less expensive supplier may have limited capacity.

If the production costs are concave, Karlin (1958) presented conditions under which a generalized $(s, S)$ policy is optimal. This policy is represented by two parameters $s$ and $S$ as well as the optimal inventory level $y(x)$, where no orders are placed if $x \geq s$ and $y$ is such that $y(u) \geq y(x) \geq S \geq s$ when $u \leq x \leq s$. Basically, this policy tends to place large orders when the inventory level is lower thereby, benefiting from economies of scale associated with the concave costs. Porteus (1971) considered the special case where the costs are concave and piecewise linear (consider a case where there is a setup cost) and showed that there exist finite break points $s_{1} \leq \ldots s_{n} \leq S_{n} \leq \ldots \leq S_{1}$ such that the ordering policy is to order up to $S_{1}$ if $x<s_{1}$; order up to $S_{2}$ if $s_{1} \leq x<s_{2}$ and so on, and do not order if $x \geq s_{n}$. Porteus (1991) provides detailed examples and explanations for the piecewise linear convex and concave costs.

\subsection{Multiple Period Dynamic Model}

The single period models studied in the previous section are applicable only in very limited settings such as products that sell in one season, perishable products and products at the end of their life cycle. Most of the other settings require analysis of the inventory system over multiple periods. There are two types of models that are studied in this context - finite horizon and infinite horizon. In the finite horizon models, there are a fixed number of periods and the objective is to minimize the discounted total expected costs over the horizon. In the finite horizon models, the objective could be either to minimize the discounted expected costs or the average expected costs over the infinite horizon. Another source of differentiation is related to whether unsatisfied demand is lost or backlogged.

The demand in every period is assumed to be independent and identically dis- 
tributed. In addition, the costs are assumed linear and stationary in order to obtain nice structures on the optimal policy. The sequence of events in every period is similar to the single period model in that orders are placed at the beginning of the period; demand is observed during the period; maximum demand is satisfied at the end of the period and resulting costs are incurred. The assumption is that the lead time is negligible so that the orders placed are available at the end of the period. The finite horizon problem with backlogging can be formulated as follows. Let $J_{n}(x)$ be the optimal cost to go given that there are $n$ periods remaining in the horizon and $x$ is the on-hand inventory.

$$
J_{n}(x)=\min _{y \geq x}\left\{c(-x)+G_{n}(y)+\alpha \int_{0}^{\infty} J_{n-1}(y-\xi) d F(\xi)\right.
$$

Note that in the above formulation, we assume that the terminal costs are zero but one could add salvage costs at the end of the horizon. Since the single period cost function $G(y)$ is convex in $y$, one can use recursion to show that the objective is convex in $y$ and hence there exists an optimal base stock level $y^{*}$ in each period. In the lost-sales case the cost to go recursion is given as follows and a similar analysis can be done.

$$
J_{n}(x)=\min _{y \geq x}\left\{c(-x)+G_{n}(y)+\alpha\left(\int_{0}^{y} J_{n-1}(y-\xi) d F(\xi)+\int_{y}^{\infty} J_{n-1}(0) d F(\xi)\right)\right.
$$

A more compact representation of the above problem can be presented by creating a function $v(y, \xi)$ given by

$$
v(y, \xi)= \begin{cases}a(y-\xi) & \xi \leq y \\ b(y-\xi) & \xi \geq y\end{cases}
$$

and utilizing that in the cost to go function.

$$
J_{n}(x)=\min _{y \geq x}\left\{c(-x)+G_{n}(y)+\alpha\left(\int_{0}^{\infty} J_{n-1}(v(y, \xi)) d F(\xi)\right.\right.
$$

Clearly, if $a=1, b=1$ it represents backlogging; if $a=1, b=0$ it represents lost sales and if $a=0, b=0$ we have an example of perishable goods. Now define 
$M(y)=G(y)-\int_{0}^{\infty} \alpha \cdot c \cdot v(y, \xi) d F(\xi)$. Let $y_{n}$ be the optimal base stock for period $n$. Then

$$
J_{n}(x)=c(-x)+G_{n}\left(y_{n}\right)+\alpha\left(\int_{0}^{\infty} J_{n-1}\left(v\left(y_{n}, \xi\right)\right) d F(\xi)\right)=-c x+\sum_{i=1}^{n} \alpha^{n-i} M_{n}\left(y_{n}\right)
$$

Veinott (1965b) showed that the above transformation enables one to simplify the problem associated with finding the optimal base stock inventory levels for each period because they are the $y_{n}$ values that minimize $M_{n}\left(y_{n}\right)$ which depends only on the parameters and expected cost when $n$ periods are remaining. This solution is also called the myopic solution since we need to solve only for the current period. Veinott (1965b) presented general conditions under which the myopic solution is optimal for the finite and infinite $(\alpha<1)$ horizon discounted cost problem. The optimal myopic solution depends on whether demand is backlogged or not. In the case of backlogging the optimal inventory is given by

$$
y^{*}=F^{-1}\left(\frac{\pi-c+\alpha c}{\pi+h}\right)
$$

\subsubsection{Lead Time}

In a dynamic inventory model, the concept of lead time becomes important. Karlin and Scarf (1958) showed that if the lead time from the supplier is fixed $L$ and all demands are backlogged then that problem could be converted into an equivalent single period problem with some adjustments. The assumption of complete backlogging is critical here because the approach relies on keeping track of the system stock (rather than just the on-hand inventory). The basic idea is that one keeps track of inventory on-hand plus all orders that have been placed (but not received yet) minus any backlogged demand. The effect of orders placed in period $t$ are felt in period $L+t$, so the approach is to consider the total demand in the next $L+1$ periods and bring the system stock to that level. Note the critical fractile still remains the same as given in (12), what changes is the cumulative probability density function $F$ (which is now a convolution of $L+1$ demand distributions). This is easy to compute for stationary and independent normal distributions since the resulting distribution is also normal 
with $\hat{\mu}=(L+1) \mu$ and $\hat{\sigma}=\sqrt{L+1} \sigma$. The reason that backlogging assumption is important because the state space for the dynamic program can be collapsed in to a single state (that represents the system stock) rather than having a vector of $L+1$ variables which represent how much was delivery is expected in the next $L$ periods in addition to the current inventory level.

The case of stochastic lead time has been studied by several researchers. The problem arises in this case because it is difficult to compute the system stock as in the deterministic demand case because knowing when an order was placed does not help in terms of identifying when the order will arrive. Also it is statistically possible that orders that were placed later are delivered before earlier orders. Kaplan (1970) was the first one to show that under two simple assumptions, it is possible to replicate results corresponding to the deterministic case. The two assumptions that are required are - (1) later orders are not delivered before earlier orders; (2) the lead time distribution does not change due to outstanding back orders. Nahmias (1979) showed that the above assumptions are equivalent to a delivery process generated by a sequence $\left\{A_{t}\right\}$ of independent and identically distributed random variables such that if $A_{t}=k$ then all the orders placed $k$ or more periods before would be delivered in the current period. This transformation allows one to mimic the optimality of the myopic policy. Further, for the the average cost analysis, it suffices to assume identically distributed lead times and independence is not always necessary.

The lost sales model even with deterministic lead times is an open problem in terms of determining the optimal policy. Morton (1971) presents bounds for the optimal ordering policy as well as the discounted cost function for the stationary problem. The heuristics presented are myopic (or near myopic) in nature and are not necessarily base stock policies. Through a limited computational study, the author provides evidence that such heuristics may be very close to optimal. More recently, van Donselaar et. al. (1996) compare the performance of a base stock policy with another myopic heuristic and show empirically that their dynamic myopic heuristic outperforms the stationary base stock policy in a significant manner. 


\subsubsection{Setup Costs}

Scarf (1960) showed that under general conditions on the cost function (such as Kconvexity) of the one period expected cost, the $n$-period dynamic inventory problem has an optimal $(\mathrm{s}, \mathrm{S})$ policy. Iglehart (1963) considers the discounted infinite horizon problem and shows the optimality of the $(s, S)$ policy by giving bounds on sequences $\left\{s_{n}\right\}$ and $\left\{S_{n}\right\}$ and establishing their limiting behavior. He also extends this result to the case with fixed lead time. Veinott (1966b) showed the above results for a different set of conditions such as the negative of the one period expected costs are unimodal and that the absolute minima of the one period costs are rising over time. Zheng (1991) presents a simple proof for the optimality of the $(s, S)$ policy for the discounted and average infinite horizon problems by constructing an $(s, S)$ or a variant solution to the optimality equation. Although the $(s, S)$ parameters are computable for the single period problem, they are more difficult to compute for the dynamic case. Veinott and Wagner (1965) give an optimal algorithm for computing these parameters. Ehrhardt (1979) presented a heuristic for computing these parameters using a power approximation. This approximation has been shown to be very accurate under a wide variety of settings. However, these approximations suffer when the variance of the demand is very small (if setup cost is also large). Zheng and Federgruen (1991) provide an efficient algorithm to compute these values.

\subsubsection{Capacity Constraint}

In most realistic environments, it is not possible for the firm to produce (order) as much as required because of production or storage capacity. This poses fundamental problems in the analysis. Wijngaard (1972) introduces the notion of a modified base stock policy where a firm tries to produce as much as possible (if unable to reach the base stock). As indicated in Federgruen and Zipkin (1986ab), Wijngaard (1972) addresses the optimality and (non)-optimality of this policy for finite and infinite horizon problems under restrictive assumptions. In a series of two papers, Federgruen and Zipkin (1986ab) show that a modified base stock policy is optimal for the discounted multiple period and discounted and average cases in the infinite horizon under general conditions such as when the expected single period cost is convex and 
a discrete demand distribution. Although the optimality of such a policy was established, it was very hard to compute the actual parameters efficiently. de Kok (1989) notes that the modified base stock policy can be computed using the fact that the inventory position at the start of a period equals $S-X$, where $X$ is the waiting time in a $\mathrm{D} / \mathrm{G} / 1$ queue. The author also provides a simple algorithm to compute the first two moments of $X$ and a heuristic for computing $S$. Tayur (1993) introduced the parallel between the dam model and the inventory dynamics equation and the used the notion of a shortfall - representing the cumulative amount of falling short of the optimal base stock level due to capacity constraint. This allows one to construct a sequence of uncapacitated infinite horizon problems that converge to the capacitated solution under consideration. Then the optimal base stock level can be easily computed. For capacitated inventory systems, infinitesimal perturbation analysis has become an efficient approach to compute (through simulation) the optimal parameter values.

\section{Alternative Demand Assumptions}

In all the discussions thus far we assumed that the demand distributions in the different periods were identical and known (hence a stationary distribution). However, in several real environments the demand distributions may be different in different periods. In this section, we highlight the key developments in those areas.

\subsection{Non-stationary Demand}

In the non-stationary demand case, the demand is assumed to be from non-identical distributions in each period. Karlin (1960a) studied the non-stationary inventory problem with zero lead time and showed that the time specific base stock policy is optimal. That is, based on the distributions of demand there is an optimal base stock level in each period. Further, he showed that if the demand distributions are stochastically increasing in different periods, then the optimal base stock levels are also increasing. Veinott (1965b) showed that if the optimal base stock levels are such that in each period one needed to place an order to get to the base stock level then 


\begin{tabular}{|l|l|c|l|}
\hline Year & Reference & Year & Reference \\
\hline 1959 & Scarf & 1997 & Aviv and Federgruen \\
\hline $1960 \mathrm{a}$ & Scarf & 1998 & Graves, Kletter and Hetzel \\
\hline $1960 \mathrm{ab}$ & Karlin & 1998 & Kapuscinski and Tayur \\
\hline 1964 & Iglehart & 1999 & Bollapragada and Morton \\
\hline $1965 \mathrm{~b}$ & Veinott & 1999 & Lariviere and Porteus \\
\hline 1972 & Hausman and Peterson & 1999 & Gavirneni and Tayur \\
\hline 1975 & Johnson and Thompson & 1999 & Gavirneni, Kapuscinski and Tayur \\
\hline 1978 & Morton & 1999 & Cheng and Sethi \\
\hline 1985 & Azoury & 2000 & Lee, So and Tang \\
\hline 1989 & Zipkin & 2000 & Scheller-Wolf and Tayur \\
\hline 1992 & Lovejoy & 2001 & Gavirneni and Tayur \\
\hline 1994 & Heath and Jackson & 2001 & Kaminsky and Swaminathan \\
\hline 1995 & Morton and Pentico & 2001 & Toktay and Wein \\
\hline 1997 & Sethi and Cheng & 2001 & Huang, Scheller-Wolf and Tayur \\
\hline 2001 & Gallego and Ozer & 2002 & Aviv \\
\hline
\end{tabular}

Table 2: Papers on Single Product Single Stage Non Stationary and Dependent Demands.

a myopic policy is still optimal for the non-stationary case. Thus, a myopic policy is optimal for the case when $y_{t}^{*} \leq y_{t+1}^{*}$ for all $t$. Further, the stationary distribution case is a special case where the identical base stock levels across the different periods imply that one would necessarily have to place an order given that the initial inventory is less than the base stock level, leading to the optimality of the myopic solution. In general when the myopic policy is not optimal it is difficult to obtain the exact optimal parameters. Morton (1978) provided a sequence of upper and lower bounds for the optimal base stock levels such that the $n t h$ bound requires the knowledge about the first $n$ demand distributions giving planning horizon results for the infinite horizon case. Lovejoy (1992) considers the non-stationary inventory problem and provides conditions and stopping rules for utilizing myopic policies under very general settings. Morton and Pentico (1995) provide myopic solutions that may be $\epsilon$ close to the optimal solution and hence, term it as near-myopic solution and test their efficacy through a detailed computational study. Gavirneni and Tayur (2001) provide a quick method to compute the base stock levels using Direct Derivative Estimation (DDE). Bollapragada and Morton (1999) study the non-stationary inventory problem with setup costs and provide a very effective myopic heuristic to the problem by ap- 
proximating the future problem in each period by a stationary problem and obtaining the solution for that problem.

\subsubsection{Cyclic Demand Schedule}

Many firms encounter a demand pattern where the demand follows a cyclic pattern in that the cycles repeat themselves after a while. For example, one could look at the demand during the four quarters in traditional industries. Karlin (1960ab) discusses the optimality of the periodic base stock policy which implies that there are different base stock levels for each of the periods, under stationary costs and cyclic non-stationary demand for the discounted infinite horizon case, and also provides an optimal algorithm. Zipkin (1989) extends the above results to the infinite horizon average cost case with both non-stationary cyclic demand and non-stationary costs. More recently, Kapuscinski and Tayur (1998) consider the capacitated version of the problem and prove the optimality of the modified periodic base stock policy for dynamic multi-period and infinite horizon (discounted and average cost) cases. They also provide an algorithm to find the optimal base stock levels using infinitesimal perturbation analysis. Independently, Aviv and Federgruen (1997) also proved the optimality of the modified periodic base stock policy. Scheller-Wolf and Tayur (2000) extend the above to include minimum order quantities and lead times.

\subsection{Bayesian Demand Updates}

In many cases, the demand distribution may not be completely known but as more information is obtained (with demand realization) the estimate of the demand distribution can be refined. Scarf $(1959,1960 a)$ introduces the bayesian demand updates where the distribution of the demand is supposed to depend on one or more parameters and those parameters are refined using bayesian updates as more information related to the demand process is obtained. In particular, he assumes that the demand distribution is gamma with an unknown scale parameter and shows the optimality of the base stock policy. Karlin (1960b) and Iglehart (1964) extended the analysis to the case where the demand distribution is of the range type between 0 and $\omega$ where $\omega$ is unknown. The fundamental assumption in this approach is that the prior for the 
parameter and the demand distribution are from the same conjugate family so that the posterior distribution has an easily workable form. Azoury (1985) extends these results to other types of distributions such as Weibull and Normal (with known and unknown mean and variance). Further, she explains how the optimal base stock levels can be determined easily. Basically, a single normalized base stock level is computed in advance and then the optimal base stock level is obtained by scaling this value. The scale factor depends on a function of the sufficient statistic of the unknown parameter that is generated based on past demand. Recently, Lariviere and Porteus (1999) extend the above observations to other environments and provide conditions under which a firm would invest in additional inventory to learn more about the demand as well as cases where despite poor sales, the product is stocked in order to obtain better information. Huang, Scheller-Wolf and Tayur (2001) use a Hidden Markov Model (HMM) to update the state of the unknown demand of a new product.

\subsection{Forecast Evolution}

Another important reality that needs to be incorporated in inventory models is the forecasting process utilized. Hausman and Peterson (1972) develop a multi-period model with terminal demand where the forecast errors get refined in a Lognormal process. In a capacitated environment they show that optimal policy is of threshold type and present heuristics to solve the problem. More recently, Kaminsky and Swaminathan (2001) consider a forecast generation process which depends on forecast bands (and the demand is expected to be uniformly spread in the interval) that get refined over time. In a terminal demand capacitated setting, they show the optimality of the threshold type policy and provide very efficient algorithm for cases with and without holding costs. Researchers have also studied the Martingale model for forecast evolution along with production-inventory decisions. Heath and Jackson (1994) and Graves et. al. (1998) independently introduce these models and develop heuristical methods to solve the problem. Recently, Toktay and Wein (2001) study this problem and use heavy traffic approximations to prove the optimality of the base stock policy under those assumptions. More recently, Aviv (2002) presents a supply chain model where different members observe subsets of the underlying demand information, and adapt their forecasting and replenishment policies accordingly. For each member, he 
identifies an associated demand evolution model, for which he proposes an adaptive inventory replenishment policy that utilizes the Kalman Filter technique. He provides a simple methodology for assessing the benefits of various types of information-sharing agreements between members of the supply chain.

\subsection{Demand Dependencies}

Demand across the different periods could be related to each other in some environments. Veinott (1965ab) through his work on non-stationary demands provides general conditions under which even a dependent demand process may have myopic solutions. Johnson and Thompson (1975) utilize those results to show that even when the demand is described by a ARMA (auto-regressive moving average) model with an additive shock, a myopic policy remains optimal under mild conditions. Sobel (1988) provided general conditions under which a myopic solution remains optimal.

Another way to represent the dependencies is to assume that the demand gets generated from a Markov process, so that the state in the current period affects the demand in the next period. Karlin and Fabens (1960) introduced a Markovian demand model and postulate that a state dependent $(s, S)$ policy would be optimal. However, they restrict themselves to stationary $(s, S)$ policy due to complexity. Sethi and Cheng (1997) prove the optimality of the state dependent $(s, S)$ policy for markovian demand for both finite and infinite horizon problems. They can also extend the model to capture periods with no orders as well as capacity and service constraint. Gavirneni and Tayur (1999) consider a modified version of the Markovian process which may be generated due to a fixed ordering schedule ("Target Reverting") at the customer end. They prove the optimality of a modified base stock policy and provide computational results. Gallego and Ozer (2001) study a situation where customers may place orders in advance (more common in a make to order environment) which provides the firm with advance demand information. They show that state-dependent $(s, S)$ and base-stock policies are optimal for stochastic inventory systems with and without fixed costs. The state of the system reflects the knowledge of advance demand information. They also determine conditions under which advance demand information has no operational value. 
Another reason for demand dependencies is that the firm may be receiving orders from another firm that may be following some optimal policy such as $(s, S)$. Gavirneni et. al. (1999) study the value of this additional demand information in a capacitated multi-period setting. Lee, So and Tang (2000) study the value of information in a two level supply chain with non-stationary end demand and show that value of information could be very high, particularly in cases where the demand may be correlated over time. Demand is also affected by the pricing decisions used by firms. Cheng and Sethi (1999) consider a general model where the customer demand is generated by a Markov process whose state is dependent on the promotion decisions. They assume a fixed cost for promotion and that the demand moves to a stochastically higher state the next period. The firm tries to find the optimal promotion schedule in terms of which periods to promote and what inventory levels to stock. For a finite horizon problem they show that there exists a threshold level $\mathrm{P}$ such that if the initial inventory is greater than $\mathrm{P}$ then it is optimal to promote, and also show that a base stock policy is optimal for the linear cost case.

\section{Generalizations}

There have been several generalizations of the single firm single product form that has been discussed so far. In this section, we will briefly explore these generalizations.

\subsection{Multi-Echelon}

The natural extension of a single firm model in a supply chain setting relates to considering multiple echelons under a single firm. Clark and Scarf (1960) study a serial system with each facility (representing an echelon) supplying the downstream facility within a deterministic non-zero lead time. The echelon stock is defined as the stock at that facility plus the stock at all the facilities downstream. The holding and stock-out costs are assessed independently, taking into account the echelon stock of each facility. Under the above assumptions, they show that the problem can be decomposed into independent problems each for one facility and that the base stock policy still remains optimal in that case where each facility tries to bring the inventory 
as close as possible to the optimal echelon base stock level. They also provide a mechanism by which the optimal base stocks can be computed by sequentially going from the last facility moving backwards.

\begin{tabular}{|c|c|c|c|}
\hline Year & Reference & Year & Reference \\
\hline \multicolumn{4}{|c|}{ Multiple Echelons } \\
\hline 1960 & Clark and Scarf & 1962 & Clark and Scarf \\
\hline 1981 & Eppen and Schrage & 1984b & Federgruen and Zipkin \\
\hline 1985 & Roundy & 1985 & Schmidt and Nahmias \\
\hline 1989 & Rosling & 1994,1995 & Glasserman and Tayur \\
\hline 1994,1998 & Chen and Zheng & 1999 & Chen \\
\hline 2000 & Parker and Kapuscincki & 2001 & Chen and Song \\
\hline 2001 & Muharremoglu and Tsitsiklis & & \\
\hline \multicolumn{4}{|c|}{ Multiple Products } \\
\hline 1963 & Hadley and Whitin & 1969 & Ignall and Veinott \\
\hline 1969 & Ignall & 1981 & Silver \\
\hline 1984 & Federgruen, Gronevelt and Tijms & $1985 \mathrm{ab}$ & Karmarkar, Kekre and Kekre \\
\hline 1987 & Karmarkar, Kekre and Kekre & 1988 & Atkins and Iyogun \\
\hline 1990 & Gallego & 1993 & Karmarkar \\
\hline 1993 & Lee and Billington & 1996 & Federgruen and Catalan \\
\hline 1996 & Lambrecht et. al. & 1997 & Lee and Tang \\
\hline 1998 & Swaminathan and Tayur & 1998 & Eynan and Kropp \\
\hline 1998 & Anupindi and Tayur & 1999 & Bollapragada and Rao \\
\hline 1999 & Bassok, Anupindi and Akella & 2001 & Rajagopalan and Swaminathan \\
\hline 2001 & Bispo and Tayur & 2002 & Swaminathan and Lee \\
\hline 2002 & Rao, Swaminathan and Zhang & 2002 & Van Mieghem and Rudi \\
\hline \multicolumn{4}{|c|}{ Multiple Suppliers } \\
\hline 1964 & Fukuda & $1966 \mathrm{a}$ & Veinott \\
\hline 1969 & Wright & 1993 & Anupindi and Akella \\
\hline 1999 & Swaminathan and Shanthikumar & 2000 & Scheller-Wolf and Tayur \\
\hline \multicolumn{4}{|c|}{ Process Randomness } \\
\hline 1958 & Karlin & 1990 & Henig and Gerchak \\
\hline 1991 & Bassok and Akella & 1994 & Ciarallo, Akella and Morton \\
\hline 1995 & Lee and Yano & & \\
\hline
\end{tabular}

Table 3: Papers on generalizations of the base case.

Federgruen and Zipkin (1984b) provide a simple method to streamline the computations in the infinite horizon case with normal demands. Chen and Song (2001) consider a multi-stage serial system with Markov modulated demand in that the demand distribution in each period is determined by the current state of an exogenous Markov chain. They show the optimality of a state dependent echelon base-stock 
policy for the long-run average costs case. They also provide an algorithm for determining the optimal base-stock levels and extend their results to serial systems in which there is a fixed ordering cost at the last stage and to assembly systems with linear ordering costs. In a more recent work, Muharremoglu and Tsitsiklis (2001) employ a novel approach based on decomposition of the problem into a series of single-item single-customer problems that enables them to provide a simpler proof for the optimality of echelon base stock policies. This approach enables them to extend their results to several variants of the problem including stochastic lead time and Markovian demand processes.

One variant of the serial system is the assembly system where more than one facility may be involved in an upstream echelon to provide parts for the downstream assembly operation. Schmidt and Nahmias (1985) study the case with two echelons where the upstream echelon has two suppliers with different deterministic lead times and a fixed assembly lead time. The optimal policy has an interesting structure in that the assembly level has a base stock policy while the policy at the upstream echelon is such that it tries to balance the echelon stock of the two components taking into account the difference in lead times of the two suppliers. Rosling (1989) showed that under certain initial conditions, an assembly system can be reduced to a serial system with modified lead times so that results due to Clark and Scarf (1960) may apply to the modified system. Another variant of the serial system is the distribution system where one facility at an upstream level supplies to multiple facilities downstream. The results of the serial system do not carry forward easily to the distribution network. Eppen and Schrage (1981) analyze a one warehouse and multiple retailer network and explore the tradeoff involved therein.

The addition of capacity restrictions on the above generalization leads to several complications. Firstly, the simple base stock policies may no longer be optimal, and secondly, even under restricted set of base stock policies the computation of these parameters is challenging. Glasserman and Tayur $(1994,1995)$ develop a solution for finding the optimal base stock levels at the different echelons under a modified base stock policy and utilize a simulation based optimization procedure using infinitesimal perturbation analysis to develop an efficient solution methodology for finding the 
optimal parameter values. They also extend their approach to the assembly and distribution networks under certain conditions. Parker and Kapuscinski (2000) demonstrate that a modified echelon base-stock policy is optimal in a two-stage system for a capacity dominating condition. They show that this holds for both stationary and non-stationary stochastic customer demand for finite and infinite horizons under discounted and average-cost criteria. There have been numerous attempts to develop a better grasp of the case with setup costs at both stages even for a serial system starting with Clark and Scarf (1962). Recently, Chen (1999) utilized the nested policy ideas developed by Roundy (1985) for deterministic systems, in a two stage serial system with Poisson demand and setup costs to develop $94 \%$ optimal policies for the problem.

\subsection{Multiple Products}

Another dimension of extension of these models is along the number of products. If all the products have independent demand and there is no capacity or production restrictions then naturally the problem can be decoupled into independent single product problems. However, in reality, a firm produces multiple products (which may be similar in functionality) and may have a common capacity to utilize for those products. Hadley and Whitin (1963) consider the capacitated news vendor problem when there is a common capacity constraint of the form

$$
\sum_{i} a_{i} y_{i} \leq b
$$

They solve the problem by relaxing the constraint and obtain an explicit expression for the optimal quantities in terms of the Lagrangean multiplier $\lambda$ as follows. If the optimal base stock levels are not feasible then

$$
y_{i}^{*}(\lambda)=F^{-1}\left\{\frac{\pi_{i}-c_{i}-a_{i} \lambda}{\pi_{i}+h_{i}}\right\}
$$

and $\lambda$ is chosen so that

$$
\sum_{i} a_{i} y_{i}^{*}(\lambda) \leq b
$$

Recently, Bispo and Tayur (2001) study base stock policies under various scenarios of capacity sharing across products in a single stage, serial and re-entrant systems. 
The notion of similarity among the different products and the fact that they could be substituted for each others demand was explored by Ignall and Veinott (1969). They showed that in fact the base stock policies are optimal for the problem with nested downward substitution (where product 1 can substitute for product $2,3, \ldots \mathrm{n}$; product 2 can substitute for product $3,4, \ldots \mathrm{n}$ and so on) in a multiple period infinite horizon setting. Bassok et. al. (1999) provide an alternative proof for the same result. The problem related to downward substitution as well as set up costs is extremely hard to obtain theoretical insights on. Rao et. al. (2002) provide a highly efficient algorithm for finding the optimal production/substitution strategy for that problem using a combination of dynamic programming and simulation based optimization.

Another important concept with multiple products relates to postponing the point of differentiation of the products in order to reduce inventory as a result of risk pooling - storing inventory of semi-finished products reduces the risk associated with stocking that inventory. Lee and Billington (1993) and Lee and Tang (1997) study postponement in the context of distribution through the channel. Swaminathan and Tayur (1998) study the postponement issue within the context of a capacitated final assembly facility and term the semi-finished products as vanilla boxes. For more details on research conducted on postponement strategies, see Swaminathan and Lee (2002).

Another complexity with multiple products is studied in the Joint Replenishment Planning (JRP) context, where there is a major setup cost at each order (across products), and a minor setup cost that may be product dependent. The stochastic version is particularly important as it is a very common problem in practice. A reasonable ordering policy that has been studied extensively are the can-order or $(s$, $c, S)$ policies. In such a policy, when any item $i$ inventory drops below its reorder point $s_{i}$ a reorder is scheduled and all other item $j$ whose inventory is below their can-order $\operatorname{limit} c_{j}$ are also included in the order. Ignall (1969) showed that can-order policies are not optimal in general. However, Silver (1981) and Federgruen et. al. (1984) have empirically shown that can-order policies perform well. Atkins and Iyogun (1988) provide a lower bound for the joint replenishment problem above and show that canorder policies are not very efficient when the joint setup cost is high. They show that a heuristic based on a periodic ordering can outperform can-order policies in a 
significant manner. Eynan and Kropp (1998) propose yet another periodic heuristic and show that is close to the optimal solution through a computational study.

Another important problem is the stochastic economic lot sizing problem (ELSP) where several items need to be produced in a common facility with limited capacity, under significant uncertainty regarding demands, production times, or combinations thereof. Gallego (1990) considers the problem of scheduling the production of several items in a single facility that can produce only one item at a time. He assumes that demands are random with constant expected rates and allows back orders and charge holding and backlogging costs at linear time weighted rates. Items are produced at continuous constant rate and setup times and setup costs are item dependent constants. He proposes a real time scheduling system that utilizes the expected demand to create the initial schedules and adjusts them for the randomness. Federgruen and Catalan (1996) propose cyclical base stock policies for the problem. Under this scheme, when the facility is assigned to a given item, production continues until either a specific target inventory level is reached or a specific production batch has been completed; different items are produced in a given sequence or rotation cycle, possibly with idle times inserted between the completion of an item's production batch and the setup for the next item. Optimal policies within this class which minimize holding, backlogging, and setup costs are effectively determined and evaluated. Bollapragada and Rao (1999) as well as Anupindi and Tayur (1998) are more recent contributions to ELSP and cyclic schedule problems.

Earlier research related to manufacturing lead times, order release and capacity releases is summarized in Karmarkar (1993). Among those Karmarkar, Kekre and Kekre $(1985 \mathrm{ab}, 1987)$ in a series of papers studied lot sizing in multi-item multimachine job shops and cellular environments. Lambrecht et. al. (1996) introduce the notion of safety lead times in queuing models of a make to order manufacturing environment. They show that there is a convex relationship of expected waiting time, variance of the waiting time and the quoted lead time as a function of the lot size and a concave relationship of the service level as a function of the lot size. This allows them to accurately quantify the safety time and to compute the associate service levels. Although lsizing and capacity expansion are very closely related 
they have not been studied extensively in integrated models. In a recent work, Rajagopalan and Swaminathan (2001) study the capacity expansion and lot sizing in a multi-product environment with deterministic known demand and present effective heuristics and bounds for the problem. More recently, Van Mieghem and Rudi(2002) develop a framework to study multi-period multi-product problems of stochastic capacity investment and inventory management. The optimal capacity and inventory decisions balance overages with underage costs. The optimal balancing conditions are interpreted as specifying multiple critical fractiles of the multivariate demand distribution; they also suggest appropriate measures for and trade-offs between product service levels. They establish dynamic optimality of inventory and capacity policies for the lost sales case.

\subsection{Multiple Suppliers}

Several firms have more than one supplier for any particular product in order to hedge against the uncertainty in the delivery process as well as to avoid being held captive by the supplier. Fukuda (1964), Veinott (1966a) and Wright (1969) study the case where rush orders from a reliable supplier could be obtained one period earlier (at an additional cost) than the normal lead time in an emergency situation. They showed that there are two base stock levels - an emergency base stock level and a normal base stock level. If the inventory level is lower than the emergency stock level then orders are placed with the more reliable supplier to get to the emergency stock level. Then additional orders are placed with the normal supplier to reach the normal base stock level. Anupindi and Akella (1993) study a different version of the problem where the lead times and their unit costs are different. They show that the optimal policy has two parameter base stock levels. If the inventory is higher than the larger base stock level then no orders are place. If it is in between the two base stock levels then orders are placed only with the less expensive supplier and else orders are placed with both. In particular, they note that orders are never placed with the more expensive supplier alone. Scheller-Wolf and Tayur (2000) extend these results into a more general model. Swaminathan and Shanthikumar (1999) showed that the above structure is driven by the continuity assumption in demand and need not hold in general for discrete demand distributions. There are several other papers 
which deal with supply contracts; see Anupindi and Bassok (1998), Lariviere (1998), Corbett and Tang (1998) and Tsay et. al. (1998) for reviews on this topic.

\subsection{Randomness in Process}

Another important generalization of the traditional inventory models relates to the randomness in production process or also called random yield. Several industries particularly semiconductors face a critical problem related to managing the yield of the process and simultaneously determining the inventory levels. Karlin (1958) explores the notion of randomness in supply by assuming a probability distribution for the the receipt of harvest from the producer. The decision is whether to order given a set of probability of possible harvests. Bassok and Akella (1991) study the joint production and ordering decisions in an environment where demand is random and amount received is a random fraction of that ordered. Henig and Gerchak (1990) consider the case where the amount received is a fraction of amount ordered and characterize the optimal policy. For the multi-period case they show the convexity of the cost function and show the existence of the optimal order point. Ciarallo et. al. (1994) consider a problem where the total capacity itself is random and show that there are optimal order points in that case as well although the convexity of the cost function is lost in multi-period and infinite horizon problems. They also present the notion of extended myopic policies in the infinite horizon case. Lee and Yano (1995) present a comprehensive literature review on random yield research.

\subsection{Approximations}

Given the difficulty of solving complex inventory problems exactly, many approximate methods for solving these problems have been proposed. One such approximation is the large deviation approximation to study capacitated systems, both in discrete time and continuous time models. The common characteristics in such systems is that inventory is held in part to compensate for the capacity restriction. The basic idea in this approximation is that if the tail of the distribution of demand is exponentially bounded, then the tail of the distribution of inventory shortfall is approximately exponential. Further, the exponent in this approximation can be easily computed. 
This approximation is useful because the performance of the inventory system with respect to service level directly depends on the tail distribution. Glasserman (1998) provides a detailed overview of this approach.

Another approximation that has been used by researchers is the one related to approximately characterize the optimal policy for a multi-echelon inventory system with economies of scale. Chen and Zheng (1994, 1998) discuss near-optimal policies (in a continuous setting) for multi-echelon inventory systems. Chen (1998) provides a detailed description of various approximations that have been considered by researchers in this area.

Finally, approximations related to results under heavy traffic assumptions have been used in inventory models as well. These approximations are useful when the load of the system is very high and utilization is close to 100\% (see Toktay and Wein 2001).

\section{Applications}

\begin{tabular}{|l|l|}
\hline Year & Reference \\
\hline 1988 & Cohen and Lee \\
\hline 1990 & Cohen, Kamesam, Kleindorfer, Lee and Tekerian \\
\hline 1993 & Lee and Billington \\
\hline 1998 & Swaminathan, Smith and Sadeh \\
\hline 2000 & Ettl, Feigin, Lin and Yao \\
\hline 2000 & Rao, Scheller-Wolf and Tayur \\
\hline 2000 & Graves and Willems \\
\hline
\end{tabular}

Table 4: Papers on Applications.

Several applications in the past years have been developed within the context of supply chain management. These applications can be clearly classified into two categories - one that builds large integrated models of a multi-tiered supply chain primarily based on deterministic assumptions about demand, supply and process and the other based on decomposition of the large scale supply chains and approximation of the behavior through the development of detailed tactical supply chain models discussed in this chapter at each of those nodes. We will focus on the latter. 
One of the first large scale model framework that linked decision and performance throughout the material-production-distribution supply chain was developed by Cohen and Lee (1988). The model structure could be used to predict the performance of a firm with respect to the cost of its products, the level of service provided to its customers and the responsiveness and flexibility of the production/distribution system. The analysis took into account the nature of the product produced, the process technologies used to manufacture the products, the structure of the facility network used to manage the material flow and the competitive environment in which the firm operates. It differed from earlier work in that a decentralized control was assumed and combined the performance of the single nodes to create the combined supply chain effect. A series of linked, approximate sub-models and a heuristic optimization procedure were developed. Each sub-model in the model framework used tractable stochastic models. A software package to support the structure was also introduced. Another large scale implementation was the development of Optimizer, a decision support for IBM's multi-echelon inventory system for managing spare parts inventory (see Cohen et. al. 1990). The model and analysis in this work relied heavily on decoupling the multi-echelon inventory system into several single level inventory systems and determining the optimal (or near-optimal) parameters for those single echelon systems. Starting from the echelon closest to the customer, the parameters are found in an iterative manner, by coupling the demands at the higher echelons of the supply chain with decisions at lower echelons regarding the inventory parameter decisions, namely the $(s, S)$ values.

Lee and Billington (1993) consider a model of the supply chain with a periodic review policy and stochastic demand that has decentralized control. The idea is similar, to analyze the performance of individual entities and then create their combined effect. This model provided various insights for supply chain planning at HP. Ettl et. al. (2000) adopt a queuing approximation to determine the service level implications in a multi-tiered network to find the optimal inventory levels to stock at different points in the supply chain. In their approach, each of the sites follows a base stock policy and they assume that there is a nominal lead time for production and transportation at each of the echelons. The actual lead times may be greater due to shortages. They model each inventory buffer as an infinite-server $\mathrm{M} / \mathrm{G} / \infty$ 
queue following a base stock policy (as in Buzzacott and Shanthikumar 1993). They assume a Poisson arrival process for demand. Using the above assumptions, they couple the service measures across the supply chain with the base stock levels chosen. Using a heuristic approach they find the optimal base stock levels. This model and approximation are validated and refined by supply chain managers through detailed simulation analysis based on an enhanced version of the supply chain library developed in Swaminathan et.al. (1998). The above model and implementation led to an estimated $\$ 750$ million reduction in inventory at IBM and was awarded the Franz Edelman Prize in 1999.

More recently, Rao et. al. (2000) describe the successful implementation of a dynamic supply chain model at Caterpillar. They analyze alternative supply chain configurations for a new product line incorporating expedited deliveries, partial backlogging of orders and sales that were responsive to service provided. Utilizing a combination of models from network flow theory, inventory management and simulation, they analyze alternative choices for the supply chain configuration. Graves and Willems (2000) develop a framework for strategic inventory placement in a supply chain that is subject to demand or forecast uncertainty. They model the supply chain as a network where each entity operates according to a base stock policy, faces bounded demand and has a guaranteed delivery lead time between the echelons. They utilize the spanning tree concept and formulate the problem as a deterministic optimization problem to obtain the safety stock. This model was utilized by product flow teams at Eastman Kodak. A more detailed description of approximations for multi-stage multi-item models appears in de Kok and Fransoo (2002), chapter 14 of this handbook.

\section{Conclusions and Future Directions}

In the Internet age as firms try to completely integrate their operations, tactical planning models for supply chain integration are becoming extremely relevant. In this chapter, we have provided an overview of several streams of research on this broad topic that have been conducted by researchers in the past. Clearly the above stream of research has had tremendous impact on both academic research as well as 
on practice. However, there are certain changes that are taking place with the advent of the Internet which have opened rich topics for new research (see Swaminathan and Tayur 2002 for details). Firstly, more and more firms are trying to integrate their production decisions with their pricing decisions and this opens up several topics of research which coordinate supply chain and pricing decisions under cooperative and competitive settings. Cachon (2002) explores some of the models developed therein. Secondly, the focus of operations in many firms is changing from a single entity optimization model to a more collaborative decision making process. Analysis needs to be conducted on models which integrate information and supply chain decisions. Chen (2002) explores such models in another chapter. Finally, there is a growing need to decision support systems that can operate in real time to provide solutions for tactical supply chain problems.

Acknowledgements: The authors wish to thank Ton de Kok and Srinagesh Gavirneni for their detailed comments on an earlier version of this chapter.

\section{References}

Anupindi R. And R. Akella, Diversification under Supply Uncertainty, Management Science, 39(8), 944-963, 1993.

Anupindi R. And Y. Bassok, Supply Contracts with Quantity Commitments and Stochastic Demand, Quantitative Models for Supply Chain Management, edited by Tayur, Ganeshan and Magazine, Kluwer Publishing, Norwell MA, 197-232, 1998.

Anupindi R. And S. Tayur, Managing Stochastic Multi Product Systems: Model, Measures and Analysis, Operations Research, S98-111, 1998.

Arrow K., T. Harris and J. MarschaK, Optimal inventory policy, Econometrica, 19, 250-272, 1951.

Arrow K., S. Karlin AND H. ScARF, Studies in the mathematical theory of inventory and production, Stanford University Press, Stanford, CA, 1958.

Atkins D. And P.O. Iyogun, Periodic versus 'Can-Order' Policies for Coordinated Multi-Item Inventory Systems, Management Science, 34(6), 791-796, 1988. 
Aviv Y., A Time Series Framework for Supply Chain Inventory Management, to appear Operations Research, 2002.

Aviv Y. And A. Federgruen, Stochastic inventory models with limited production capacity and periodically varying parameters, Probability of Engineering Information Sciences, 11, 107-135, 1997.

Azoury K., Bayes solution to dynamic inventory models under unknown demand distribution, Management Science, 31, 1150-1160, 1985.

Bassok Y. And R. Akella, Ordering and Production Decisions with Supply Quantity and Demand Uncertainty, Management Science, 37, 1556-1574, 1991.

Bassok, Y., R. Anupindi And R. Akella, Single period multiproduct inventory models with substitution, Operations Research, 47(4), 632-642, 1999.

Bispo, C. And S. Tayur, Managing Simple Re-entrant Flow Lines: Theoretical Foundation and Experimental Results,IIE Transactions, 33(8), 609-623, 2001.

Bollapragada R. and U.S. RaO, Single Stage Resource Allocation and Economic Lot Sizing, Management Science, 45(6), 889-904, 1999.

Bollapragada S. And T.E. Morton, A Simple Heuristic for Computing Nonstationary (s,S) Policies, Operations Research, 47(4), 576-584, July-August, 1999.

Buzzacott J. And J.G. Shanthikumar, Stochastic Models of Manufacturing Systems, Prentice Hall, Englewood Cliffs, NJ, 1993.

CACHON G., Supply chain coordination: pricing tactics for coordinating the supply chain, to appear, Supply Chain Management - Handbook in OR/MS, edited by Graves and de Tok, North-Holland, Amsterdam, 2002.

Chen F., On (R, NQ) Policies Serial Inventory Systems, Quantitative Models for Supply Chain Management, edited by Tayur, Ganeshan and Magazine, Kluwer Publishing, Norwell MA, 71-110, 1998.

CHen F., 94\% Effective policies for two stage serial inventory system with stochastic demand, Management Science, 12, 1679-1696, 1999.

CHEN F., Information sharing and Supply chain coordination, to appear, Supply 
Chain Management - Handbook in OR/MS, edited by Graves and de Tok, NorthHolland, Amsterdam, 2002.

Chen F. And J. Song, Optimal Policies for Multiechelon Inventory Problems with Markov-Modulated Deamnd, Operations Research, 49(2), 226-234, 2001.

Chen F. And Y. Zheng, Lower bounds for multi-echelon stochastic inventory systems, Management Science, 40, 1426-1443, 1994.

Chen F. And Y. Zheng, Near optimal echelon stock (R,Q) policies in multi-stage serial systems, Operations Research, 46(4), 592-602, 1998.

Cheng F. And S.P. Sethi, A periodic review inventory model with demand influenced by promotion decisions, Management Science, 45, 1510-1523, 1999.

Ciarallo F., R. Akella and T.E. Morton, A periodic review production planning model with uncertain capacity, Management Science, 40, 320-332, 1994.

Clark A. and H. Scarf, Optimal Policies for a multi-echelon inventory problem, Management Science, 6, 475-490, 1960.

Clark A. And H. ScArf, Approximate solutions to a simple multi-echelon inventory problem, Studies in Applied Probability and Management Science, edited by Arrow and Scarf, Stanford University Press, Stanford, CA, 88-110, 1962.

Cohen M.A., P.V. Kamesam, P. Kleindorfer, H.L. Lee and A. Tekerian, Optimizer: IBM's Multi-Echelon Inventory System for Managing Service Logistics, Interfaces, 20(1), 65-82, 1990.

Cohen M.A. And H.L. Lee, Strategic Analysis of Integrated Production Distribution Systems, Operations Research, 34(4), 482-499, 1988.

Corbett C.J. And C.S. TAng, Designing Supply Contracts: Contract Type and Information Asymmetry, Quantitative Models for Supply Chain Management, edited by Tayur, Ganeshan and Magazine, Kluwer Publishing, Norwell MA, 269-298, 1998.

Debodt M. And S.C. Graves, Continuous review policies for a multi-echelon inventory problem with stochastic demand, Management Science, 31, 1286-1299, 1985. DE KOK T., A moment-iteration method for approximating the waiting time charac- 
teristics of the GI/G/1 queue, Probability of Engineering and Information Sciences, 3, 273-287, 1989.

De Kok T. And J. Fransoo, Supply Chain Operations: Comparing Planning Concepts, to appear, Supply Chain Management - Handbook in OR/MS, edited by Graves and de Tok, North-Holland, Amsterdam, 2002.

Edgeworth F., The mathematical theory of banking, Journal of Royal Statistics Society, 51, 113-127, 1888.

Ehrhardt F., (s,S) policies for a dynamic inventory model with stochastic lead times, Operations Research, 32, 121-132, 1984.

EHRHARDT R., The power approximation for computing $(\mathrm{s}, \mathrm{S})$ inventory policies, Management Science, 25, 777-786, 1979.

Eppen G. And L. Schrage, Centralized Ordering Policies in a Multi-Warehouse System with Lead Times and Random Demand, Multi-Level Production Inventory Control Systems: Theory and Practice (ed. L.B. Schwartz), North Holland, Amsterdam, 51-58, 1981.

Ettl M., G. Feigin, G. Lin And D.D. YaO, A supply network model with basestock control and service requirements, Operations Research, 48(2), 216-232, 2000.

Eynan A. And D. Kropp, Periodic review and joint replenishment in stochastic demand environments, IIE Transactions, 30(11), 1025-1033, 1998.

Federgruen A., H. Groenevelt and H.C. Tijms, Coordinated Replenishment in a Multi-Item Inventory System with Compound Poisson Demand, Management Science, 30, 344-357, 1984.

Federgruen A. And Z. Catalan, Stochastic economic lot scheduling problem: cyclical base stock policies with idle times, Management Science, 42(6), 783-796, 1996.

Federgruen A. And P. Zipkin, An efficient algorithm for computing optimal (s,S) policies, Operations Research, 32, 818-832, 1984a.

Federgruen A. And P. Zipkin, Approximation of dynamic multi-location produc- 
tion inventory problem, Management Science, 30, 69-84, 1984b.

Federgruen A. And P. Zipkin, An inventory model with limited production capacity and uncertain demands I: The average cost criterion, Mathematics of Operations Research, 11, 193-207, 1986a.

Federgruen A. AND P. ZiPkin, An inventory model with limited production capacity and uncertain demands II: The discounted cost criterion, Mathematics of Operations Research, 11, 208-215, 1986b.

FUKUDA Y., Optimal policies for inventory problems with negotiable lead time, Management Science, 10, 690-708, 1964.

Gallego G., Scheduling the Production of Several Items with Random Demands in a Single Facility, Management Science, 36, 1579-1592, 1990.

Gallego G. And O. Ozer, Integrating Replenishment Decisions With Advance Demand Information, Management Science, 47, 1344-1360, 2001.

Gavirneni S., R. Kapuscinski and S. Tayur, Value of information in capacitated supply chains, Management Science, 45, 16-24, 1999.

GAVIRnEni S. AND S. TAYUR, Managing a customer following a target reverting policy, Manufacturing and Service Operations Management, 1, 157-173, 1999.

Gavirneni S. And S. TAYur, An Efficient Procedure for Non-stationary Inventory Control, IIE Transactions, 33(2), 83-89, 2001.

Glasserman P., Service Levels and Tail Probabilities in Multistage Capacitated Production Inventory Systems, Quantitative Models for Supply Chain Management, edited by Tayur, Ganeshan and Magazine, Kluwer Publishing, Norwell MA, 41-70, 1998.

Glasserman P. AND S. TAYUR, The stability of a capacitated multi-echelon production inventory system under a base stock policy, Operations Research, 42, 913-925, 1994.

Glasserman P. And S. TAYur, Sensitivity analysis for base stock levels in multiechelon production inventory system, Management Science, 41, 263-281, 1995. 
Graves S., D.B. Kletter And W.B. Hetzel, A Dynamic Model for Requirements Planning with Application to Supply Chain Optimization, Operations Research, 46, S35-49, 1998.

Graves S., A.H.G. Rinnoy Kan and P. Zipkin, Handbook in OR/MS on Logistics of Production and Inventory, North-Holland, Vol. 4, Amsterdam, Netherlands, 1993.

Graves S. And S. Willems, Optimizing strategic safety stock placement in supply chains, Manufacturing and Service Operations Management, 2(1), 2000.

Hadley G. And T. Whitin, Analysis of Inventory Systems, Prentice-Hall, Englewood Cliffs, NJ, 1963.

Hausman W.H., Sequential Decision Problems: A Model to Exploit Existing Forecasters, Management Science, 16(2), B93-B110, 1969.

Hausman W.H. And R. Peterson, Multiproduct Production Scheduling for Style Goods with Limited Capacity, Forecast Revisions and Terminal Delivery, Management Science, 18(7), 370-383, 1972.

Heath D.C. And P.L. JaCKson, Modeling the Evolution of Demand Forecasts with Application to Safety Stock Analysis in Production/Distribution Systems, IIE Transactions, 26(3), 17-30, 1994.

Henig M. And Y. Gerchak, The structure of periodic review policies in the presence of random yield, Operations Research, 38, 634-643, 1990.

Heyman D. And M. Sobel, Stochastic models in operations research, vol. II, McGraw-Hill, New York, 1984.

Huang P., Scheller-Wolf A. And S. Tayur, Dynamic Capacity Partitioning During New Product Introduction, GSIA Working Paper, Carnegie Mellon University, 2001.

IGLEHART D., Optimality of $(\mathrm{s}, \mathrm{S})$ policies in the infinite horizon dynamic inventory problem, Management Science, 9, 259-267, 1963.

IGLEHART D., The dynamic inventory model with unknown demand distribution, 
Management Science, 10, 429-440, 1964.

Ignall E., Optimal Continuous Review Policies for Two Product Inventory Systems with Joint Setup Costs, Management Science, 15, 277-279, 1969.

IGNALl E. AND A. VeInotT, Optimality of myopic inventory policies for several substitute products, Management Science, 15, 284-304, 1969.

Johnson G. And H. Thompson, Optimality of myopic inventory policies for certain dependent demand process, Management Science, 21, 103-1307, 1975.

Kaminsky P. And J.M. Swaminathan, Utilizing Forecast Band Refinement for Capacitated Production Planning, Manufacturing and Service Operations Management, 3(1), 68-81, 2001.

KAPlan R., A dynamic inventory model with stochastic lead times, Management Science, 16, 491-507, 1970.

KapUscinski R. AND S. TAYUR, A capacitated production inventory model with periodic demand, Operations Research, 1998.

KarLin S., One-stage inventory models with uncertainty, Studies in the Mathematical Theory of Inventory and Production (edited by K. Arrow, S. Karlin and H. Scarf), Stanford University Press, Stanford, CA, 1958.

KARLIN S., Dynamic inventory policy with varying stochastic demands, Management Science, 6, 231-258, 1960a.

KaRLIN S., Optimal policy for dynamic inventory process with stochastic demands subject to seasonal variations, Journal of Society of Industrial Applied Mathematics, 8, 611-629, 1960b.

Karlin S. And A. Fabens, The $(\mathrm{s}, \mathrm{S})$ Inventory Model under Markovian Demand Process, Mathematical Methods in the Social Sciences, edited by Arrow, Karlin and Suppes, Stanford University Press, Stanford, CA, 159-175, 1960.

KARLIN S. AND H. SCARF, Inventory models of the Aroow-Harris-Marschak type with time lag, Studies in the Mathematical Theory of Inventory and Production, edited by Arrow, Karlin and Scarf, Stanford University Press, Stanford, CA, 1958. 
Karmarkar U.S., Manufacturing Lead Times, Handbook in OR/MS on Logistics of Production and Inventory, (edited by S.Graves, A.H.G. Rinnoy Kan and P.H. Zipkin), 287-321, 1993.

Karmarkar U.S., S. KeKre and S. Kekre, Lot Sizing in Multi-Item MultiMachine Job Shops, IIE Transactions, 17, 290-292, 1985a.

Karmarkar U.S., S. Kekre And S. Kekre, Lot Sizing and Lead Time Performance in a Manufacturing Cell, Interfaces, 15, 1-9, 1985b.

Karmarkar U.S., S. Kekre and S. Kekre, The Dynamic Lot Sizing Problem with Startup and Reservation Costs, Operations Research, 35(3), 389-398, 1987.

Lambrecht, S. Chen And N.J. Vandaele, A lot sizing model with queuing delays: The issue of safety time, European Journal of Operational Research, 89, 269276, 1996.

Lariviere M. A., Supply Chain Contracting and Coordination with Stochastic Demand, Quantitative Models for Supply Chain Management, edited by Tayur, Ganeshan and Magazine, Kluwer Publishing, Norwell MA, 233-268, 1998.

Lariviere M. A. AND E. Porteus, Stalking information: Bayesian inventory management with unobserved lost sales, Management Science, 45(3), 346-353, 1999.

Lee H.L. And C. Billington, Materials Management in Decentralized Supply Chains, Operations Research, 41(5), 835-847, 1993.

Lee H.L. And C. TAng, Modelling the Costs and Benefits of Delayed Product Differentiation, Management Science, 43(1), 40-53, 1997.

Lee H.L., R. So And C.S. TAng, The Value of Information in a Two Level Supply Chain, Management Science, 46(5), 626-643, 2000.

LeE H.L. And C.A. YAno, Lot sizing with random yields: a review, Operations Research, 43(2), 311-334, 1995.

LOVEJOY W., Stopped myopic policies in some inventory models with generalized demand processes, Management Science, 38, 688-707, 1992.

Muharremoglu A. And J. Tsitsiklis, Echelon Base Stock Policies in Uncapac- 
itated Serial Inventory Systems, Working Paper, MIT, 2001.

MorTon T., The near-myopic nature of the lagged proportional cost inventory problem with lost sales, Operations Research, 19, 1708-1716, 1971.

Morton T., The non-stationary infinite horizon inventory problem, Management Science, 24, 1474-1482, 1978.

Morton T. And D. Pentico, The finite horizon non-stationary stochastic inventory problem, Management Science, 41, 334-343, 1995.

Nahmias S., Simple approximations for a variety of dynamic lead time lost sales inventory models, Operations Research, 27, 904-924, 1979.

PARKer R. AND R. KAPuscinski, Optimal Inventory Policies for a Capacitated Two Echelon System, Working Paper, University of Michgan, 2000.

Porteus E., On the optimality of generalized (s,S) policies, Management Science, 17, 411-427, 1971.

Porteus E., The optimality of generalized $(\mathrm{s}, \mathrm{S})$ policies under uniform demand densities, Management Science, 18, 644-646, 1972.

Porteus E., Stochastic Inventory Theory, Stochastic Models - Handbook in OR/MS, edited by Heyman and Sobel, North-Holland, Amsterdam, 605-652, 1991.

Rajagopalan S. And J.M. Swaminathan, A Coordinated Production Planning Model with Capacity Expansion and Inventory Management, Management Science, 47(11), 1562-1580, 2001.

RaO, U.S., J.M. Swaminathan And J. Zhang, Multi-Product Inventory Planning with Downward Substitution, Stochastic Demand and Setup Costs, to appear, IIE Transactions, 2002.

Rao U.S., A. Scheller-Wolf And S. Tayur, Development of a rapid response supply chain at caterpillar, Operations Research, 48(2), 189-204, 2000.

Rosling K., Optimal inventory policies for assembly systems under random demands, Operations Research, 37, 565-579, 1989.

Roundy R., $98 \%$ effective integer ratio lot sizing for one warehouse multi retailer 
systems, Management Science, 31, 1416-1430, 1985.

SCARF H., Bayes solution of the statistical inventory problem, Annals of Mathematical Statistics, 30, 490-508, 1959.

SCARF H., The optimality of $(\mathrm{s}, \mathrm{S})$ policies in dynamic inventory problem, Mathematical Methods in the Social Sciences (edited by K. Arrow, S. Karlin and P. Suppes), Stanford University Press, Stanford, CA, 1960.

SCARF H., Some remarks on the Bayes solution to the inventory problem, Naval Research Logistics Quarterly, 7, 591-596, 1960a.

Scheller-Wolf A. AND S. TAyur, A Markovian Dual Source Production-Inventory System with Order Bands, GSIA Working Paper, Carnegie Mellon University, 2000.

Schmidt C. And S. Nahmias, Optimal policy for a two stage assembly system under random demand, Operations Research, 33, 1130-1145, 1985.

Sethi S.P. And F. Cheng, Optimality of $(\mathrm{s}, \mathrm{S})$ policies in inventory models with markovian demand, Operations Research, 45, 931-939, 1997.

Silver E., Establishing Reorder Points in the (S,c,s) Coordinated Control System under Compound Poisson Demand, International Journal of Production Research, 9, 743-750, 1981.

Sobel M., Dynamic affine logistics models, Technical Report, SUNY, Stony Brook, 1988.

Swaminathan J.M. And H.L. Lee, Design for Postponement, to appear, Supply Chain Management - Handbook in OR/MS, edited by Graves and de Tok, NorthHolland, Amsterdam, 2002.

Swaminathan J.M., S.F. Smith and N. Sadeh, Modelling supply chain dynamics: A multi-agent approach, Decision Sciences, 29(3),607-632, 1998.

Swaminathan J.M. And S. TAYUR, Managing broader product lines through delayed differentiation using vanilla boxes, Management Science, 44, S161-72, 1998.

Swaminathan J.M. And S. Tayur, Models for Supply Chains in E-Business, Working Paper, The Kenan-Flagler Business School, University of North Carolina, Chapel 
Hill, 2002.

Swaminathan J.M. And J.G. Shanthikumar, Supplier Diversification: The Effect of Discrete Demand, Operations Research Letters, 24(5), 213-221, 1999.

TAYUR S., Computing the optimal policy in capacitated inventory models, Stochastic Models, 9, 1993.

Tayur S., R. Ganeshan and M. Magazine, Quantitative Models for Supply Chain Management, Kluwer Academic Publishers, Norwell, MA, 1998.

Toktay L.B. And L.M. Wein, Analysis of a Forecasting Production Inventory system with Stationary Demand, Management Science, 47(9), 1268-1281, 2001.

Tsay A.A., S. Nahmias and N. Agarwal, Modeling Supply Chain Contracts: A Review, Quantitative Models for Supply Chain Management, edited by Tayur, Ganeshan and Magazine, Kluwer Publishing, Norwell MA, 299-336, 1998.

van Donselaar K., T. De Kok and W. Rutten, Two replenishment strategies for the lost sales inventory model: A comparison, International Journal of Production Economics, 46-47, 285-295, 1996.

Van Mieghem J. And N. Rudi, Newsvendor Networks: Inventory Management and Capacity Investments with Discretionary Activities, to appear, Manufacturing and Service Operations Management, 2002.

Veinott A. JR., Optimal policy for a multi-product dynamic non-stationary inventory problem, Management Science, 12, 206-222, 1965a.

Veinott A. JR., Optimal policy in a dynamic single product non-stationary inventory model with several demand classes, Operations Research, 13, 776-778, 1965b.

Veinott A. JR., The status of mathematical inventory theory, Management Science, $12,745-777,1966 \mathrm{a}$

VeinotT A. JR., On the optimality of the $(\mathrm{s}, \mathrm{S})$ inventory policies: New conditions and a new proof, SIAM Journal of Applied Mathematics, 14, 1067-1083, 1966b.

Veinott A. JR. And H. WAGner, Computing optimal $(\mathrm{s}, \mathrm{S})$ inventory policies, Management Science, 11, 525-552, 1965. 
WiJngaARD J., An inventory problem with constrained order capacity, TH-Report 72-WSK-63, Eindhoven University of Technology, 1972.

Wright G., Optimal ordering policies for inventories with emergency ordering, $O p$ erations Research Quarterly, 20, 111-123, 1969.

ZHENG Y., A simple proof for optimality of $(\mathrm{s}, \mathrm{S})$ policies in infinite horizon inventory systems, Journal of Applied Probability, 28, 802-810, 1991.

Zheng Y. And A. Federgruen, Finding optimal (s, S) policies is about as simple as evaluating a single policy, Operations Research, 39(4), 654-665, 1991.

ZIPKIN P., Critical number policies for inventory models with periodic data, Management Science, 35, 71-80, 1989.

ZIPKIN P., Foundations of Inventory Management, McGraw Hill, Boston, 2000. 